Groups Geom. Dyn. 5 (2011), 189-206

DOI $10.4171 / \mathrm{GGD} / 122$
Groups, Geometry, and Dynamics

(C) European Mathematical Society

\title{
The action of a nilpotent group on its horofunction boundary has finite orbits
}

\author{
Cormac Walsh*
}

\begin{abstract}
We study the action of a nilpotent group $G$ with finite generating set $S$ on its horofunction boundary. We show that there is one finite orbit associated to each facet of the polytope obtained by projecting $S$ into the torsion-free component of the abelianisation of $G$. We also prove that these are the only finite orbits of Busemann points. To finish off, we examine in detail the Heisenberg group with its usual generators.
\end{abstract}

Mathematics Subject Classification (2010). 20F65, $20 \mathrm{~F} 18$.

Keywords. Group action, horoball, max-plus algebra, metric boundary, Busemann function.

\section{Introduction}

Given a group acting by isometries on a space, one often finds it useful to study the induced action on some boundary of the space, for example the hyperbolic boundary of a Gromov hyperbolic space or the ideal boundary of a CAT(0) space. The hope is that the action on the boundary is simpler than the action on the space itself.

Of course, we may endow any finitely-generated group with its word metric, and then the group acts isometrically on itself by left translations. One can then study the induced action on the horofunction boundary, which exists for any metric space.

The case of $\mathbb{Z}^{d}$ with an arbitrary finite generating set was investigated by Rieffel [6] in connection with his work on quantum metric spaces. Essential to his work was that the action on the horoboundary is amenable and that there are sufficiently many finite orbits. In [4], Develin studied in greater detail the horoboundary of $\mathbb{Z}^{d}$ and the action of $\mathbb{Z}^{d}$ on it.

In this paper, we study the case of a finitely-generated nilpotent group. We find that, just as in the case of $\mathbb{Z}^{d}$, there are always finite orbits, whatever the generating set.

To state our results, we need the following construction. Recall that the quotient $G /[G, G]$ of a group $G$ by its commutator subgroup is an abelian group, called the

\footnotetext{
*This work was partially supported by the joint RFBR-CNRS grant number 05-01-02807.
} 
abelianisation. If $G$ is finitely generated, then so is the abelianisation, which can therefore be written as a direct product $Z_{0} \times \mathbb{Z}^{N}$, where $Z_{0}$ is a finite abelian group (the torsion subgroup) and $N \in \mathbb{N}$. So we can define a projection map $\phi$ assigning to every element of $G$ its equivalence class in $(G /[G, G]) / Z_{0}$. We consider $\mathbb{Z}^{N}$ to be embedded in $\mathbb{R}^{N}$. Let $P:=\operatorname{conv}(\phi(S))$ be the convex hull of the image of the generating set $S$ under the map $\phi$.

We will assume for simplicity that $S$ is symmetric, that is, $S=S^{-1}$, since this makes the word length distance a metric. The results should also be true, however, in the more general case.

A particularly interesting subset of the horofunction boundary is its set of Busemann points. These are closely related to the geodesic paths. In general, all geodesic paths converge to Busemann points [6]. Moreover, when the metric is integer valued, as in the present setting, the Busemann points are precisely the limits of the geodesic paths.

Recall that a facet of a polytope is a proper face of maximal dimension, in other words, of co-dimension 1.

Theorem 1.1. Let $G$ be a nilpotent group with finite symmetric generating set $S$ and consider the action of $G$ on its horofunction boundary coming from the word length metric associated to $S$. Then, there is a natural one-to-one correspondence between the finite orbits of Busemann points and the facets of $P$.

When $G=\mathbb{Z}^{d}$, the map $\phi$ is just the identity map. In this case, it was shown in [4] that all boundary points are Busemann and that there is a one-to-one correspondence between the orbits and the proper faces of $P$, with the finite orbits corresponding to the facets. We will give an example later of a non-abelian nilpotent group where this stronger correspondence breaks down.

We also have more precise results for the discrete Heisenberg group, which has the following presentation:

$$
H_{3}:=\langle a, b \mid[[a, b], a]=[[a, b], b]=e\rangle .
$$

We use the word length metric coming from the usual set of generators $S:=$ $\left\{a, b, a^{-1}, b^{-1}\right\}$. For this group with these generators, an exact formula for the word length metric is known. We show directly that there are four finite orbits of Busemann points, each consisting of a single horofunction. These horofunctions are the limits of the four geodesic words $a^{\epsilon_{a}} b^{\epsilon_{b}} a^{\epsilon_{a}} b^{\epsilon_{b}} \ldots$, with $\epsilon_{a}= \pm 1$ and $\epsilon_{b}= \pm 1$. We also show that every other Busemann point is the limit of a geodesic path of the form $t \rightarrow c^{m} b^{n} a^{ \pm t}$ or $t \rightarrow c^{m \pm l t} b^{ \pm t} a^{l}$. Using the formula for the metric, all these Busemann points can be calculated explicitly; see Theorems 5.3 and 5.4. 


\section{The horofunction boundary}

The horofunction boundary of a proper metric space $(X, d)$ is defined as follows. One assigns to each point $z \in X$ the function $\psi_{z}: X \rightarrow \mathbb{R}$,

$$
\psi_{z}(x):=d(x, z)-d(b, z),
$$

where $b$ is some base-point. The map $\psi: X \rightarrow C(X), z \mapsto \psi_{z}$, defines a continuous injection of $X$ into $C(X)$, the space of continuous real-valued functions on $X$ endowed with the topology of uniform convergence on compact sets. The horofunction boundary is defined to be

$$
X(\infty):=\operatorname{cl}\left\{\psi_{z} \mid z \in X\right\} \backslash\left\{\psi_{z} \mid z \in X\right\},
$$

and its elements are called horofunctions. This definition seems to be due to Gromov [5]. For more information, see [2], [6], and [1].

The action of the isometry group $\operatorname{Isom}(X, d)$ of $X$ extends continuously to an action by homeomorphisms on the horofunction compactification. One can check that

$$
g \cdot \xi(x)=\xi\left(g^{-1} \cdot x\right)-\xi\left(g^{-1} \cdot b\right),
$$

for any isometry $g$, point $x$, and horofunction $\xi$.

The metric spaces in which we will be interested in this paper are the finitelygenerated nilpotent groups with their word length metrics. Recall that the Cayley graph of a group $G$ with symmetric generating set $S$ has a vertex for each element of $G$ and an edge between two vertices $u$ and $v$ if $u^{-1} v \in S$. The word length distance $d(u, v)$ between $u$ and $v$ in $G$ is the length of the shortest path between the corresponding vertices in the Cayley graph. By construction, it is a left invariant metric. We choose the identity element $e$ as the base-point.

A path is a sequence of group elements. We say that a path is geodesic if it is an isometry from $\mathbb{N}$ to $G$. It is known that geodesic paths converge to points in the horofunction boundary. In the present setting, since the metric is integer valued, we may define a Busemann point as the limit of a geodesic. See [6] and [1] for the definition in more general spaces.

For any finite word $w$ with letters in $S$, we denote by $\bar{w}$ the element of $G$ obtained by multiplying the letters of $w$, and we say that $w$ represents $\bar{w}$. We denote by $|w|$ the length of $w$, that is, its number of letters. We say that a finite word $w$ is a geodesic word if $|w|=d(e, \bar{w})$, and that an infinite word is geodesic if each of its finite prefixes is. Obviously, there is a one-to-one correspondence between geodesic paths and pairs $(g, w)$, where $g$ is in $G$ (the starting point of the path) and $w$ is an infinite geodesic word. The steps of a geodesic path are the letters of the corresponding geodesic word. Continuing to use terminology of [4], we say that the direction of a geodesic path is the set of letters that occur infinitely often as steps.

We start off with an easy proposition telling us when two geodesics converge to the same Busemann point. Note that the proof relies on the fact that the horofunctions are integer valued. 
Proposition 2.1. Let $\gamma_{1}$ and $\gamma_{2}$ be two geodesics in a finitely-generated group. The following are equivalent:

(i) $\gamma_{1}$ and $\gamma_{2}$ converge to the same Busemann point;

(ii) for all $N \in \mathbb{N}$, there is a geodesic agreeing with $\gamma_{1}$ up to time $N$ that has a point in common with $\gamma_{2}$ and eventually agrees with $\gamma_{1}$ again;

(iii) there is a geodesic having infinitely many points in common with both $\gamma_{1}$ and $\gamma_{2}$.

Moreover, the above statements hold if

(iv) $\gamma_{1}$ and $\gamma_{2}$ have a point in common, and, for all $N \in \mathbb{N}$, there is a geodesic agreeing with $\gamma_{1}$ up to time $N$ with the same limit as $\gamma_{2}$, and a geodesic agreeing with $\gamma_{2}$ up to time $N$ with the same limit as $\gamma_{1}$

Proof. The implications (ii) $\Longrightarrow$ (iii) and (iii) $\Longrightarrow$ (i) are obvious.

Assume (i) holds, that is, $\gamma_{1}$ and $\gamma_{2}$ converge to some horofunction $\xi$. Let $N \in \mathbb{N}$. We can find $t_{2}$ large enough that

$$
\xi\left(\gamma_{2}(0)\right)-\xi\left(\gamma_{1}(N)\right)=d\left(\gamma_{2}(0), \gamma_{2}\left(t_{2}\right)\right)-d\left(\gamma_{1}(N), \gamma_{2}\left(t_{2}\right)\right) .
$$

We can then find $t_{1}$ large enough that

$$
\xi\left(\gamma_{2}\left(t_{2}\right)\right)-\xi\left(\gamma_{1}(N)\right)=d\left(\gamma_{2}\left(t_{2}\right), \gamma_{1}\left(t_{1}\right)\right)-d\left(\gamma_{1}(N), \gamma_{1}\left(t_{1}\right)\right) .
$$

Since $\gamma_{2}$ is a geodesic,

$$
\xi\left(\gamma_{2}(0)\right)=d\left(\gamma_{2}(0), \gamma_{2}\left(t_{2}\right)\right)+\xi\left(\gamma_{2}\left(t_{2}\right)\right) .
$$

It follows that

$$
d\left(\gamma_{1}(N), \gamma_{1}\left(t_{1}\right)\right)=d\left(\gamma_{1}(N), \gamma_{2}\left(t_{2}\right)\right)+d\left(\gamma_{2}\left(t_{2}\right), \gamma_{1}\left(t_{1}\right)\right) .
$$

So there exists a geodesic agreeing with $\gamma_{1}$ up to time $N$, passing through $\gamma_{2}\left(t_{2}\right)$, and agreeing with $\gamma_{1}$ after time $t_{1}$. This establishes (ii).

Now assume that (iv) holds. So there is a geodesic $\gamma_{3}$ agreeing with $\gamma_{1}$ up to any given time $N$ with the same limit as $\gamma_{2}$. Applying the equivalence of (i) and (ii) just proved, we see there is a geodesic $\gamma_{4}$ agreeing with $\gamma_{1}$ up to time $N$ and agreeing with $\gamma_{2}$ after some time $M$. A symmetrical argument shows that there is a geodesic $\gamma_{5}$ agreeing with $\gamma_{2}$ up to time $M$ and eventually agreeing with $\gamma_{1}$. It is easy to prove that the path agreeing with $\gamma_{4}$ up to time $M$ and thereafter agreeing with $\gamma_{5}$ is a geodesic satisfying the requirements of (ii).

\section{Preliminaries on nilpotent groups}

We use $[a, b]$ to denote the commutator $a^{-1} b^{-1} a b$ of two elements of a group. Also, for any two subgroups $A$ and $B$, we denote by $[A, B]$ the subgroup generated by all 
$[a, b]$ with $a \in A$ and $b \in B$. For any group $G$, the subgroup $[G, G]$ is called the commutator subgroup. It is always normal.

Recall that a group $G$ is nilpotent if $\Gamma_{n}$ is trivial for some $n \in \mathbb{N}$, where $\Gamma_{i} ; i \in \mathbb{N}$ is the lower central series defined by $\Gamma_{1}:=G$ and $\Gamma_{i+1}:=\left[\Gamma_{i}, G\right]$ for $i \in \mathbb{N}$.

We will need the following two lemmas concerning finite-index subgroups of nilpotent groups. They have similar proofs, which rely on the identities

$$
[x, y z]=[x, z][x, y][[x, y], z]
$$

and

$$
[x y, z]=[x, z][[x, z], y][y, z] .
$$

Lemma 3.1. Let $H$ be a finite index subgroup of a finitely generated nilpotent group $G$. Then, $[H, H]$ has finite index in $[G, G]$.

Proof. We use an inductive argument. The induction hypothesis is that there is a finite set $R_{i} \subset G$ such that any $g \in[G, G]$ can be expressed as $g=h^{\prime} r^{\prime} \bmod \Gamma_{i}$, with $h^{\prime} \in[H, H]$ and $r^{\prime} \in R_{i}$.

The case when $i=2$ is trivial.

Now assume the hypothesis is true for some $i \geq 2$. The assumption of the lemma implies that, for each generator $s \in S$, there is some $n_{s} \geq 1$ and $h_{s} \in H$ such that $s^{n_{s}}=h_{s}$. Let $C_{i}$ denote the set of simple commutators of weight $i$ of elements of the generating set $S$. In other words, $C_{1}:=S$ and

$$
C_{j+1}:=\left\{[c, s] \mid c \in C_{j} \text { and } s \in S\right\} \text { for } j \geq 1 .
$$

Let $c:=\left[\ldots\left[s_{1}, \ldots\right], s_{i}\right]$ be an arbitrary element of $C_{i}$. Using the identities (1) and (2), one can show that

$$
c^{n_{s_{1}} \ldots n_{s_{i}}}=\left[\ldots\left[s_{1}^{n_{s_{1}}}, \ldots\right], s_{i}^{n_{s_{i}}}\right] \bmod \Gamma_{i+1}=\left[\ldots\left[h_{s_{1}}, \ldots\right], h_{s_{i}}\right] \bmod \Gamma_{i+1} .
$$

So, letting $p_{c}:=n_{s_{1}} \ldots n_{s_{i}}$, we see that $c^{p_{c}}$ is in $[H, H]$, modulo $\Gamma_{i+1}$.

As $\Gamma_{i} / \Gamma_{i+1}$ is abelian and generated by the elements of $C_{i} \bmod \Gamma_{i+1}$ we can write any $d \in \Gamma_{i}$ in the form

$$
d=\prod_{c \in C_{i}} c^{m_{c}} \bmod \Gamma_{i+1},
$$

where the product is taken in any fixed order and $m_{c} \in \mathbb{Z}$ for all $c \in C_{i}$. Therefore, from what we have just proved,

$$
d=h \prod_{c \in C_{i}} c^{q_{c}} \bmod \Gamma_{i+1}
$$

for some $h \in[H, H]$, where $q_{c}$ is the remainder when $m_{c}$ is divided by $p_{c}$. Let $R$ be the finite set

$$
R:=\left\{\prod_{c \in C_{i}} c^{q_{c}} \mid 0 \leq q_{c}<p_{c} \text { for all } c \in C_{i}\right\} .
$$


By the induction hypothesis, there is a finite set $R_{i} \subset G$ such that any $g \in[G, G]$ can be expressed as $g=h^{\prime} r^{\prime} d$, with $h^{\prime} \in[H, H], r^{\prime} \in R_{i}$, and $d \in \Gamma_{i}$. So $g=h^{\prime} d r^{\prime} \bmod \Gamma_{i+1}$. By the result of the previous paragraph, $d=h r \bmod \Gamma_{i+1}$ for some $h \in[H, H]$ and $r \in R$. Therefore, $g=h^{\prime} h r r^{\prime} \bmod \Gamma_{i+1}$, and so the induction hypothesis is true for $i+1$.

Lemma 3.2. Let $H$ be a subgroup of a finitely generated nilpotent group $G$, and let $\Theta$ be the natural homomorphism from $G$ to its abelianisation $G /[G, G]$. If $\Theta(H)$ has finite index in $\Theta(G)=G /[G, G]$, then $H$ has finite index in $G$.

Proof. We use again an inductive argument. The induction hypothesis here is that there is a finite set $R^{\prime} \subset G$ such that any $g \in G$ can be expressed as $g=h^{\prime} r^{\prime}$ mod $\Gamma_{i}$, with $h^{\prime} \in H$ and $r^{\prime} \in R^{\prime}$.

By assumption, this is true for $i=2$.

Now assume it is true for some $i \geq 2$. The assumption of the lemma implies that, for each generator $s \in S$, there is some $n_{s} \geq 1$ and $h_{s} \in H$ such that $\Theta\left(s^{n_{s}}\right)=\Theta\left(h_{s}\right)$. Therefore, for all $s \in S$, there exists $g_{s} \in[G, G]$ such that $s^{n_{s}}=h_{s} g_{s}$.

As in the proof of the previous lemma, let $C_{i}$ denote the set of simple commutators of weight $i$ of elements of $S$, and consider an arbitrary element $c:=\left[\ldots\left[s_{1}, \ldots\right], s_{i}\right]$. Using the identities (1) and (2), one can show as before that

$$
c^{p_{c}}=\left[\ldots\left[s_{1}^{n_{s_{1}}}, \ldots\right], s_{i}^{n_{s_{i}}}\right] \bmod \Gamma_{i+1}=\left[\ldots\left[h_{s_{1}}, \ldots\right], h_{s_{i}}\right] \bmod \Gamma_{i+1},
$$

where $p_{c}:=n_{s_{1}} \ldots n_{s_{i}}$.

It follows just as before that any $d \in \Gamma_{i}$ can be written as $d=h r \bmod \Gamma_{i+1}$ for some $h \in H$ and $r \in R$, where $R$ is the finite set

$$
R:=\left\{\prod_{c \in C_{i}} c^{q_{c}} \mid 0 \leq q_{c}<p_{c} \text { for all } c \in C_{i}\right\} .
$$

By the induction hypothesis there is a finite set $R^{\prime} \subset G$ such that any $g \in G$ can be expressed as $g=h^{\prime} r^{\prime} d$, with $h^{\prime} \in H, r^{\prime} \in R^{\prime}$, and $d \in \Gamma_{i}$. So $g=h^{\prime} d r^{\prime} \bmod$ $\Gamma_{i+1}$. But we have seen that $d=h r \bmod \Gamma_{i+1}$ for some $h \in H$ and $r \in R$, and therefore, $g=h^{\prime} h r r^{\prime} \bmod \Gamma_{i+1}$. This completes the induction step.

\section{Facets and finite orbits}

Let $G$ be a nilpotent group generated by a finite symmetric set $S$. Recall that $\phi$ is the projection onto the torsion-free component of the abelianisation of $G$, and that $P$ is the convex hull of $\phi(S)$, considered as a subset of $\mathbb{R}^{N}$.

Lemma 4.1. Let $V$ be a subset of $S$ such that $\phi(V)$ is contained in a facet of $P$. Then, any word with letters in $V$ is geodesic. 
Proof. Let $F \subset \mathbb{R}^{n}$ be the facet into which $V$ is mapped, and let $f: \mathbb{R}^{n} \rightarrow \mathbb{R}$ be the linear functional defining $F$, that is, such that $f(x)=1$ for $x \in F$. So, if $z_{0} z_{1} \ldots$ is a word with letters in $V$, then $f \circ \phi\left(\bar{z}_{0} \ldots \bar{z}_{n-1}\right)=n$ for all $n \in \mathbb{Z}$. Let $y_{0} y_{1} \ldots$ be a word with letters in $S$ such that $\bar{y}_{0} \ldots \bar{y}_{m-1}=\bar{z}_{0} \ldots \bar{z}_{n-1}$ for some $n$ and $m$ in $\mathbb{Z}$. Since $f(x) \leq 1$ for $x \in P$, we have $m \geq f \circ \phi\left(\bar{y}_{0} \ldots \bar{y}_{m-1}\right)=n$. It follows that $z_{0} z_{1} \ldots$ is a geodesic word.

Lemma 4.2. Let $H$ be a nilpotent group generated as a group by a finite set $V$. Then, for any $g \in H$, we can write $g \bar{x}=\bar{y}$, where $x$ and $y$ are words with letters in $V$.

Proof. We will show the result is true for each of the groups $H / \Gamma_{i} ; i \geq 2$, using induction.

It is clearly true for the group $H / \Gamma_{2}=H /[H, H]$, since this group is abelian.

Now assume the result is true for $H / \Gamma_{i}$ for some $i \geq 2$.

Let $C_{j}$ denote the set of simple commutators of weight $j$ of elements of $V$. In other words, $C_{1}:=V$ and

$$
C_{j+1}:=\left\{[c, s] \mid c \in C_{j} \text { and } s \in V\right\} \text { for } j \geq 1 .
$$

From the induction hypothesis, for every $c \in C_{i-1}$, there exists words $x_{c}$ and $y_{c}$ with letters in $V$ such that $c \bar{x}_{c}=\bar{y}_{c} \bmod \Gamma_{i}$. For each $c \in C_{i-1}$, let $g_{c} \in \Gamma_{i}$ be such that

$$
c \bar{x}_{c}=\bar{y}_{c} g_{c} \text {. }
$$

Consider the word

$$
w:=\prod_{c \in C_{i-1}, s \in V}\left(y_{c} s^{n(c, s)} x_{c}\right)\left(x_{c} s^{m(c, s)} y_{c}\right),
$$

where the product is taken in any fixed order, and $n(c, s)$ and $m(c, s)$ are non-negative integers for all $c \in C_{i-1}$ and $s \in V$. Clearly, the letters of $w$ are in $V$.

Using (3) and the fact that $[c, h]$ and $g_{c}$ are central in $H / \Gamma_{i+1}$ for every $c \in C_{i-1}$ and $h \in H$, we get

$$
\begin{aligned}
\bar{y}_{c} s^{n(c, s)} \bar{x}_{c} & =c \bar{x}_{c} g_{c}^{-1} s^{n(c, s)} c^{-1} \bar{y}_{c} g_{c} \bmod \Gamma_{i+1} \\
& =\bar{x}_{c}\left[c, \bar{x}_{c}\right] s^{n(c, s)}[c, s]^{n(c, s)} \bar{y}_{c} \bmod \Gamma_{i+1} .
\end{aligned}
$$

Similarly,

$$
\bar{x}_{c} s^{m(c, s)} \bar{y}_{c}=\bar{y}_{c}\left[\bar{x}_{c}, c\right] s^{m(c, s)}[s, c]^{m(c, s)} \bar{x}_{c}, \quad \bmod \Gamma_{i+1} .
$$

So

$\bar{w}:=\prod_{c \in C_{i-1}, s \in V}[c, s]^{n(c, s)-m(c, s)} \prod_{c \in C_{i-1}, s \in V}\left(\bar{x}_{c} s^{n(c, s)} \bar{y}_{c}\right)\left(\bar{y}_{c} s^{m(c, s)} \bar{x}_{c}\right) \bmod \Gamma_{i+1}$. 
Since the group $\Gamma_{i} / \Gamma_{i+1}$ is generated by $C_{i} \bmod \Gamma_{i+1}$, and is in the center of $H / \Gamma_{i+1}$, any $h \in \Gamma_{i}$ can be written $\left(\bmod \Gamma_{i+1}\right)$ as the first factor in the right-hand-side of the above equation, with an appropriate choice of $n$ and $m$. The second factor can be represented by a word $z$ with letters in $V$. So $h \bar{z}=\bar{w} \bmod \Gamma_{i+1}$.

Now let $g \in H$. By the induction hypothesis, $g \bar{x}=\bar{y} \bmod \Gamma_{i}$ for some words $x$ and $y$ with letters in $V$. In other words, $g \bar{x} h=\bar{y}$ for some $h \in \Gamma_{i}$. As we have just seen, $h \bar{z}=\bar{w} \bmod \Gamma_{i+1}$ for some words $z$ and $w$ with letters in $V$. So $g \bar{x} \bar{w}=\bar{y} \bar{z}$ mod $\Gamma_{i+1}$. This completes the induction step.

We are now ready to construct geodesic paths leading to the Busemann points lying in finite orbits.

Let $F$ be a facet of $P$, and let $V$ be the set of generators mapped into $F$ by $\phi$. We denote by $\langle V\rangle$ the subgroup of $G$ generated as a group by the elements of $V$. Of course, $\langle V\rangle$ is also nilpotent. So, by Lemma 4.2, for each $g \in\langle V\rangle$, there exist words $x_{g}$ and $y_{g}$ with letters in $V$ such that $g \bar{x}_{g}=\bar{y}_{g}$. Let $C_{i}$ be the set of simple commutators of weight $i$ of elements of $V$. Take a finite word $w_{F}$ with letters in $V$ such that $x_{c}$ and $y_{c}$ appear as subwords, for every $c \in \bigcup_{i} C_{i}$. The infinite word $w_{F} w_{F} \ldots$ is geodesic, by Lemma 4.1 . We define $\Lambda_{F}: \mathbb{N} \rightarrow G$ to be the geodesic path starting at the identity, having steps given by this word. Since $\Lambda_{F}$ is geodesic, it converges to a Busemann point of the horoboundary, which we denote by $\xi_{F}$.

Lemma 4.3. Let $F$ be a facet of $P$, and let $V$ be the set of generators mapped into $F$ by $\phi$. Then, for any $g \in\langle V\rangle$, there is an $n \in \mathbb{N}$ such that $g \bar{w}_{F}^{n}$ can be written as a product of elements of $V$.

Proof. Let $\Gamma_{i}(\langle V\rangle)$ denote the descending central series of $\langle V\rangle$, and let $C_{i}$ denote the set of simple commutators of weight $i$ of elements of $V$.

We consider the groups $\langle V\rangle / \Gamma_{i}(\langle V\rangle) ; i \geq 1$ and use induction.

The case when $i=1$ is trivial.

Now assume that $g \bar{w}_{F}^{n}$ can be written $\left(\bmod \Gamma_{i}(\langle V\rangle)\right)$ in the desired form. That is to say, $g \bar{w}_{F}^{n}=\bar{v} c$ for some word $v$ with letters in $V$ and some $c \in \Gamma_{i}(\langle V\rangle)$. We know that $c$ can be written as a product of simple commutators and their inverses:

$$
c=c_{1}^{\epsilon_{1}} \ldots c_{m}^{\epsilon_{m}} \bmod \Gamma_{i+1}(\langle V\rangle),
$$

where $m \in \mathbb{N}$, and $c_{j} \in C_{i}$ and $\epsilon_{j}= \pm 1$ for all $j \in\{1, \ldots, m\}$. Recall that $\Gamma_{i}(\langle V\rangle) / \Gamma_{i+1}(\langle V\rangle)$ is in the center of $\langle V\rangle / \Gamma_{i+1}(\langle V\rangle)$. Suppose that $\epsilon_{j}=1$ for some $j$. Recall that $w_{F}$ has subwords $x_{c_{j}}$ and $y_{c_{j}}$ satisfying $c_{j} \bar{x}_{c_{j}}=\bar{y}_{c_{j}}$. So by replacing the subword $x_{c_{j}}$ in $w_{F}$ by $y_{c_{j}}$, we obtain a word with letters in $V$ that represents $c_{j} \bar{w}_{F} \bmod \Gamma_{i+1}(\langle V\rangle)$. Similarly, for any $j$ for which $\epsilon_{j}=-1$, we replace $y_{c_{j}}$ by $x_{c_{j}}$ to obtain a word with letters in $V$ representing $c_{j}^{-1} \bar{w}_{F} \bmod \Gamma_{i+1}(\langle V\rangle)$. We concatenate all these words to get a word $u$ with letters in $V$ representing $c \bar{w}_{F}^{m}$ mod $\Gamma_{i+1}(\langle V\rangle)$. So $v u$ represents $g \bar{w}_{F}^{n+m} \bmod \Gamma_{i+1}(\langle V\rangle)$. This concludes the induction step. 
Lemma 4.4. Let $F$ be a facet of $P$, and let $V$ be the set of generators mapped into $F$ by $\phi$. Then, the horofunction $\xi_{F}$ is invariant under the subgroup $\langle V\rangle$.

Proof. Let $g \in\langle V\rangle$ and $n \in \mathbb{N}$. Since $g\left[g, \bar{w}_{F}^{n}\right] \in\langle V\rangle$, we can find, by Lemma 4.3, $m \in \mathbb{N}$ such that $g\left[g, \bar{w}_{F}^{n}\right] \bar{w}_{F}^{m}$ is represented by some word $v$ with letters in $V$.

Similarly, we can find $l \in \mathbb{N}$ such that $\left[\bar{w}_{F}^{n+m}, g\right] g^{-1} \bar{w}_{F}^{l}$ is represented by a word $v^{\prime}$ with letters in $V$.

We consider the path $\gamma$ starting at the identity with steps given by the word $w_{F}^{n} v v^{\prime} w_{F} w_{F} \ldots$ By Lemma 4.1, it is a geodesic. It agrees with the geodesic path $\Lambda_{F}$ up to time $n\left|w_{F}\right|$. But $\bar{w}_{F}^{n} \bar{v}=g \bar{w}_{F}^{n+m}$, and so $\gamma$ has a point in common with the path $g \Lambda_{F}$ at time $\left|w_{F}^{n} v\right|$. We also have that $\bar{w}_{F}^{n} \bar{v} \bar{v}^{\prime}=\bar{w}_{F}^{n+m+l}$. Therefore, $\gamma$ agrees with $\Lambda_{F}$ after time $\left|w_{F}^{n} v v^{\prime}\right|$.

We now use Proposition 2.1 to deduce that $\Lambda_{F}$ and $g \Lambda_{F}$ converge to the same limit $\xi_{F}$.

Theorem 4.5. Let $F$ be a facet of $P$, and let $V$ be the set of generators mapped into $F$ by $\phi$. Then, the stabiliser of $\xi_{F}$ is $\langle V\rangle$.

Proof. We have already seen in Lemma 4.4 that $\langle V\rangle$ stabilises $\xi_{F}$.

Let $g \in G$ be such that $g \xi_{F}=\xi_{F}$. Consider the geodesics $\Lambda_{F}$ and $g \Lambda_{F}$. Since they have the same limit $\xi_{F}$, there exists a geodesic $\gamma$ having infinitely many points in common with each, by Proposition 2.1. So there exist $t_{0}, t_{1}$, and $t_{2}$ in $\mathbb{N}$ with $t_{0} \leq t_{1} \leq t_{2}$, such that $\gamma\left(t_{0}\right)=\Lambda_{F}\left(s_{0}\right), \gamma\left(t_{1}\right)=g \Lambda_{F}\left(s_{1}\right)$, and $\gamma\left(t_{2}\right)=\Lambda_{F}\left(s_{2}\right)$ for some $s_{0}, s_{1}$, and $s_{2}$ in $\mathbb{N}$. Let $f: \mathbb{R}^{n} \rightarrow \mathbb{R}$ be the linear functional defining the facet $F$, so that $f(x)=1$ for $x \in F$ and $f(x)<1$ for $x \in P \backslash F$. Since the steps of $\Lambda_{F}$ lie in $V$, we have

$$
f \circ \phi\left(\Lambda_{F}\left(s_{2}\right)\right)-f \circ \phi\left(\Lambda_{F}\left(s_{0}\right)\right)=s_{2}-s_{0} .
$$

But since $\gamma$ is a geodesic, $s_{2}-s_{0}=t_{2}-t_{0}$. We conclude that

$$
f \circ \phi\left(\gamma\left(t_{2}\right)\right)-f \circ \phi\left(\gamma\left(t_{0}\right)\right)=t_{2}-t_{0}
$$

Using the fact that $f \circ \phi$ is a homomorphism and that $f \circ \phi(x) \leq 1$ for $x \in S$, we get that every step $x$ of $\gamma$ between $t_{0}$ and $t_{2}$ satisfies $f \circ \phi(x)=1$, that is, they all lie in $V$.

Consider the finite path that starts at the identity, is identical to $\Lambda_{F}$ up to time $s_{0}$, follows $\gamma$ from $\gamma\left(t_{0}\right)=\Lambda_{F}\left(s_{0}\right)$ to $\gamma\left(t_{1}\right)=g \Lambda_{F}\left(s_{1}\right)$, and then follows $g \Lambda_{F}$ backwards along to $g$. The steps of this path lie entirely within $V \cup V^{-1}$. We deduce that $g \in\langle V\rangle$.

Corollary 4.6. For every facet $F$ of $P$, the Busemann point $\xi_{F}$ lies in a finite orbit. 
Proof. Recall that $Z_{0}$ is the torsion subgroup of the abelianisation of $G$. Choose a subset $R$ of $G$ containing one representative of each equivalence class in $Z_{0}$.

Since $F$ is a facet of $P$, the set of vectors $\phi(V)$ generates a finite index subgroup of $\mathbb{Z}^{N}$. So there is a finite subset $R^{\prime}$ of $G$ such that, for any $g \in G$, there exists $r^{\prime} \in R^{\prime}$ and $v \in\langle V\rangle$ satisfying $\phi(g)=\phi(v) \phi\left(r^{\prime}\right)$.

So $\Theta(g)=\Theta\left(v r^{\prime}\right) \bmod Z_{0}$, where $\Theta$ is the natural homomorphism from $G$ to its abelianisation $G /[G, G]$. This is equivalent to $\Theta(g)=\Theta(v) \Theta\left(r r^{\prime}\right)$ for some $r \in R$. We conclude from this that $\Theta(\langle V\rangle)$ has finite index in $\Theta(G)$. So, by Lemma 3.2, $\langle V\rangle$ has finite index in $G$. But, by the theorem, $\langle V\rangle$ is exactly the stabiliser of $\xi_{F}$, and so this Busemann point has a finite orbit.

We have just shown that to every facet of $P$ there corresponds a finite orbit of Busemann points. To prove the correspondence in the opposite direction, we will need the following lemma. Note that its hypothesis that the orbit is finite is not needed in the abelian setting [4].

Lemma 4.7. Suppose the limit of a geodesic path $\gamma$ lies in a finite orbit. Then, the projection map $\phi$ maps the direction of $\gamma$ into some facet of $P$.

Proof. Let $u_{0} u_{1} \ldots$ be the geodesic word giving the steps of $\gamma$. For each $n \in \mathbb{N}$, let $\gamma^{n}$ be the geodesic starting at the identity and having steps given by the word $u_{n} u_{n+1} \ldots$. The limit of each $\gamma^{n}$ is in the same orbit as that of $\gamma$, which we have assumed finite. Therefore, there is some subsequence of geodesics $\gamma^{n_{i}}$ all of whose limits are equal. Call the common limit $\xi$.

For each $i \in \mathbb{N}$, define the finite word $w_{i}:=u_{n_{i}} \ldots u_{n_{i+1}-1}$, and let $W:=\left\{w_{i} \mid\right.$ $i \in \mathbb{N}\}$ be the set of all these words. Fix $i \in \mathbb{N}$. Since $\gamma^{n_{i}}$ is eventually just a copy of $\bar{w}_{i} \gamma^{n_{i+1}}$ shifted in time, these two geodesics have the same limit. But both $\gamma^{n_{i}}$ and $\gamma^{n_{i+1}}$ converge to $\xi$. Therefore, $w_{i}$ represents a group element in the stabiliser of $\xi$.

Now consider an infinite concatenation $z:=z_{0} z_{1} \ldots z_{m} \ldots$ of words $z_{m} ; m \in \mathbb{N}$, each in $W$. Given any $m \in \mathbb{N}$, the word $z$ can be written

$$
z=z_{0} \ldots z_{m-1} u_{n_{i}} \ldots u_{n_{i+1}-1} z_{m+1} \ldots
$$

because $z_{m}=w_{i}$ for some $i \in \mathbb{N}$. Since $p:=\bar{z}_{0} \ldots \bar{z}_{m-1}$ is in the stabiliser of $\xi$, we have $\xi(p g)=\xi(p)+\xi(g)$ for all $g \in G$. So

$$
\xi\left(\bar{z}_{0} \ldots \bar{z}_{m-1} \bar{u}_{n_{i}} \ldots \bar{u}_{n_{i}+l-1}\right)=\xi\left(\bar{z}_{0} \ldots \bar{z}_{m-1}\right)+\xi\left(\bar{u}_{n_{i}} \ldots \bar{u}_{n_{i}+l-1}\right),
$$

for $l \in\left\{0, \ldots, n_{i+1}-n_{i}\right\}$. But since $\gamma^{n_{i}}$ is a geodesic converging to $\xi$, we have $\xi\left(\bar{u}_{n_{i}} \ldots \bar{u}_{n_{i}+l-1}\right)=-l$. Since this reasoning is valid for any $m \in \mathbb{N}$, we conclude that $\xi$, evaluated along the path $\lambda$, decreases at every step, where $\lambda$ is the path starting at the identity with steps given by the word $z$. It follows from this that $\lambda$ is a geodesic. We have thus shown that every concatenation of words from $W$ is a geodesic word. 
Take now sufficiently many words $z_{0}, \ldots, z_{n-1}$ from $W$ that every generator in the direction of $\gamma$ appears in at least one. Let $a:=\phi\left(\bar{z}_{0} \ldots \bar{z}_{n-1}\right)$, and let $b$ be the intersection of the line $0 a$ in $\mathbb{R}^{N}$ with the boundary of $P$. Of course, $b$ has rational coordinates and lies in some facet $F$ of $P$. So, by Lemma 7 of [4], $b$ may be expressed as an affine combination of the vertices of $F$, with non-negative rational coefficients. Since $a$ is a rational multiple of $b$, we may express $a$ in the same way. Multiplying through by the common denominator, we can find a positive integer $l$ as large as we wish, such that $l a$ is a linear combination of the vertices of $F$ with non-negative integer coefficients. So there exists a word $x$ with letters in $\phi^{-1}(F) \cap S$ such that $\phi(\bar{x})=l a$.

Recall that $Z_{0}$ is the torsion subgroup of the abelianisation $G /[G, G]$. Let $T \subset G$ be such that there is exactly one element of $T$ in each of the equivalence classes in $Z_{0}$. Of course, $|T|=\left|Z_{0}\right|<\infty$.

We can now write $\left(\bar{z}_{0} \ldots \bar{z}_{n-1}\right)^{l}=\bar{x} t c$, with $t \in T$ and $c \in[G, G]$.

We have assumed that the stabiliser of $\xi$, which we now call $U$, has finite index in $G$. Therefore, by Lemma 3.1, $[U, U]$ has finite index in $[G, G]$. So we can write $c=r u$, with $r$ in some finite subset $R$ of $G$, and $u$ in $[U, U]$. Because $U$ stabilises $\xi$, the restriction of $\xi$ to $U$ is a homomorphism into $\mathbb{Z}$. In particular, $\xi\left(u^{-1}\right)=0$. So, for $i \in \mathbb{N}$ large enough, $d\left(u^{-1}, \gamma^{n_{0}}(i)\right)=d\left(e, \gamma^{n_{0}}(i)\right)=i$.

Let

$$
D:=\max \left\{d\left(e, t^{\prime} r^{\prime}\right) \mid t^{\prime} \in T \text { and } r^{\prime} \in R\right\} .
$$

Of course, $D$ is finite. We have

$$
d\left(e, \bar{x} \operatorname{tru} \gamma^{n_{0}}(i)\right) \leq d(e, \bar{x})+d(e, t r)+d\left(e, u \gamma^{n_{0}}(i)\right) \leq|x|+D+i .
$$

But $|x|=\operatorname{lf}(a)$, where $f: \mathbb{R}^{N} \rightarrow \mathbb{R}$ is the linear functional such that $f(g)=1$ for all $g \in F$. Also, since $\left(z_{0} \ldots z_{n-1}\right)^{l} w_{0} w_{1} w_{2} \ldots$ is a geodesic word,

$$
d\left(e,\left(\bar{z}_{0} \ldots \bar{z}_{n-1}\right)^{l} \gamma^{n_{0}}(i)\right)=l\left|z_{0} \ldots z_{n-1}\right|+i .
$$

Therefore $l f(a)+D \geq l\left|z_{0} \ldots z_{n-1}\right|$. Since $l$ can be made arbitrarily large, $f(a) \geq$ $\left|z_{0} \ldots z_{n-1}\right|$. We may now apply the fact that $f \circ \phi(s) \leq 1$ for every $s \in S$ to deduce that $f \circ \phi(g)=1$ for every letter $g$ in $z_{0} \ldots z_{n-1}$, which includes every letter in the direction of $\gamma$. Therefore, all these letters are mapped by $\phi$ to $F$.

Theorem 4.8. Every finite orbit of Busemann points can be written $\left\{g \xi_{F} \mid g \in G\right\}$ for some facet $F$ of $P$.

Proof. Let $\gamma_{1}$ be a geodesic converging to a point in a finite orbit. By Lemma 4.7, the image of the direction of $\gamma_{1}$ under $\phi$ lies in some facet $F$ of $P$. By, if necessary, premultiplying by an appropriate factor and removing an initial section of the path, we can find a geodesic path $\gamma$ converging to a point $\xi$ in the same orbit as the limit of $\gamma_{1}$ that starts at the identity and for which the image under $\phi$ of every step lies in $F$. Let the word $w_{F}$ be defined as above. Since $\xi$ is in a finite orbit, there exists some 
$m \in \mathbb{N}$ with $m>0$ such that $\bar{w}_{F}^{m} \xi$ is equal to $\xi$. So $\bar{w}_{F}^{m n} \xi=\xi$ for all $n \in \mathbb{N}$. Let $z_{0} z_{1} \ldots$ be the geodesic word giving the steps of $\gamma$, and let $\gamma_{2}$ be the geodesic path starting at the identity and having steps given by $w_{F}^{m n} z_{0} z_{1} \ldots$ for some large $n \in \mathbb{N}$. By Lemma 4.1, $\gamma_{2}$ is a geodesic. Also, $\gamma_{2}$ agrees with $\Lambda_{F}$ up to time $n m\left|w_{F}\right|$, which can be made as large as one likes. Finally, $\gamma_{2}$ converges to $\xi$, and so has the same limit as $\gamma$.

We have proved one half of condition (iv) of Proposition 2.1. To prove the other, take $n$ as large as you want and let $g:=\bar{z}_{0} \ldots \bar{z}_{n}$. Clearly, $g^{-1} \in\langle V\rangle$, where $V$ is the set of generators mapped into $F$ by $\phi$. Therefore, by Lemma 4.3, there is some $m \in \mathbb{N}$ such that $g^{-1} \bar{w}_{F}^{m}$ can be represented by some word $v$ with letters in $V$. By Lemma 4.1, the path starting at the identity and having steps given by $z_{0} \ldots z_{n} v w_{F} w_{F} \ldots$ is a geodesic. This path agrees with $\gamma$ up to time $n$, and it eventually agrees with $\Lambda_{F}$ because $\bar{z}_{0} \ldots \bar{z}_{n} \bar{v}=\bar{w}_{F}^{m}$.

It now follows from Proposition 2.1 that $\gamma$ and $\Lambda_{F}$ have the same limit, in other words, that $\xi=\xi_{F}$.

We finish this section by showing how the correspondence in the abelian setting between proper faces of $P$ and orbits of Busemann points breaks down for general finitely generated nilpotent groups.

Example. Consider the following group of nilpotency class 3:

$$
G:=\langle a, b \mid[a, g]=[b, g]=[a, h]=[b, h]=e\rangle,
$$

where $c=[a, b], g:=[a, c]$, and $h:=[b, c]$.

Any element of $G$ can be written in the form $g^{i} h^{j} c^{k} b^{l} a^{m}$, with $i, j, k, l$, and $m$ in $\mathbb{Z}$. We may take the projection map $\phi$ to be $\phi\left(g^{i} h^{j} c^{k} b^{l} a^{m}\right):=(l, m)$. The image of the generating set $\left\{a, b, a^{-1}, b^{-1}\right\}$ is a square. Let $F$ be the facet defined by the corners $\{(1,0),(0,1)\}$.

Define the word $x:=a b$. We will show that, although $x x x \ldots$ and $\Lambda_{F}$ have the same directions, their limits differ.

Lemma 4.9. Let $w$ be any word with letters in $\{a, b\}$ such that $\bar{w}=g^{m} h^{n} \bar{x}^{l}$ for some $m, n \in \mathbb{Z}$ and $l \in \mathbb{N}$. Then, $m-n$ is positive unless $w=x^{l}$.

Proof. Represent $w$ as a piecewise affine curve that starts at the origin and takes a unit step in the $x$-direction for each letter $b$, and unit step in the $y$-direction for each letter $a$. The curve will obviously finish at $(l, l)$. Write $\bar{w}$ in the form $g^{i} h^{j} c^{k} b^{l} a^{l}$. It is not hard to see that $k$ is the number of unit squares under the curve. After further thought, one also sees that $j$ is the sum of the $x$-coordinates of the upper right corners of these squares, and that $i$ is the sum of the $y$-coordinates. So the center of gravity, taking the mass of each unit square to be concentrated on its upper right corner, is $(j / k, i / k)$. We now redraw the figure with the axes rotated through a $45^{\circ}$ angle, as in Figure 1. We see that choosing $w$ to minimise $i-j$ is the same as finding the curve 


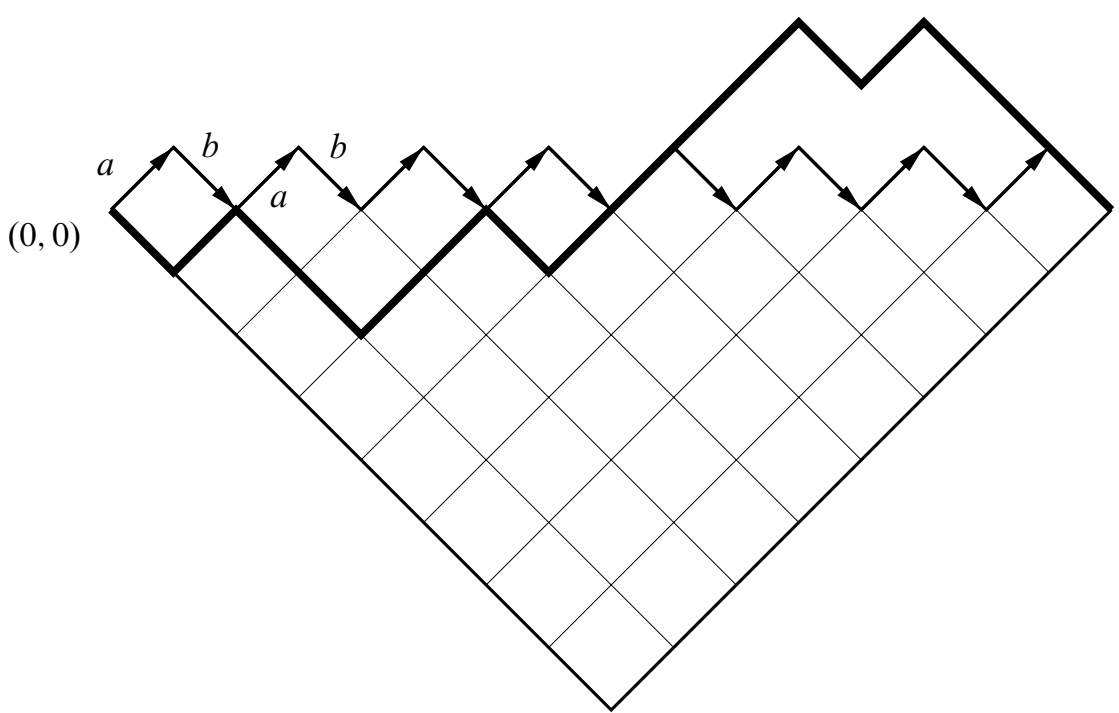

$(8,0)$

Figure 1. Diagram for the proof of Lemma 4.9.

between $(0,0)$ and $(l, l)$ having $(l+1) l / 2$ unit squares underneath it that minimises the height of the center of mass of these squares. But it is obvious that there is a unique minimising curve, namely that obtained from $x^{l}$. The conclusion follows.

Proposition 4.10. The limit $\eta$ of $x \times x \ldots$ is different from $\xi_{F}$.

Proof. Let $l \in \mathbb{N}$ and let $w$ be a geodesic word with letters in $\left\{a, b, a^{-1}, b^{-1}\right\}$ representing $g^{-1} \bar{x}^{l}$. By Lemma 4.9, either $a^{-1}$ or $b^{-1}$ occurs as a letter of $w$. Also, the excess of $a$ s over $a^{-1} \mathrm{~s}$ in $w$ equals $l$, as does the excess of $b$ s over $b^{-1}$ s. Therefore, $d\left(e, g^{-1} \bar{x}^{l}\right)=|w|>2 l$. We conclude that

$$
\eta(g)=\lim _{l \rightarrow \infty}\left(d\left(g, \bar{x}^{l}\right)-d\left(e, \bar{x}^{l}\right)\right)>0 .
$$

However, $\{a, b\}$ generates $G$, and so $\xi_{F}$ is a fixed point by Theorem 4.5 . So $\xi_{F}$ is a homomorphism from $G$ to $\mathbb{Z}$. In particular, $\xi_{F}(g)=0$. Therefore, $\eta$ and $\xi_{F}$ differ. 


\section{The boundary of the discrete Heisenberg group}

The discrete Heisenberg group $\mathrm{H}_{3}$ is the group of $3 \times 3$ upper triangular matrices of the form

$$
\left(\begin{array}{lll}
1 & x & z \\
0 & 1 & y \\
0 & 0 & 1
\end{array}\right) \quad \text { with } x, y, z \text { in } \mathbb{Z} .
$$

It is the simplest non-abelian nilpotent group. Let

$$
a:=\left(\begin{array}{lll}
1 & 1 & 0 \\
0 & 1 & 0 \\
0 & 0 & 1
\end{array}\right), \quad b:=\left(\begin{array}{ccc}
1 & 0 & 0 \\
0 & 1 & 1 \\
0 & 0 & 1
\end{array}\right), \quad \text { and } \quad c:=\left(\begin{array}{ccc}
1 & 0 & 1 \\
0 & 1 & 0 \\
0 & 0 & 1
\end{array}\right) .
$$

Observe that $a b=b a c$, that is, $c$ is the commutator of $a$ and $b$, and that the center of $\mathrm{H}_{3}$ is the cyclic group generated by $c$. The following is a presentation of $\mathrm{H}_{3}$ :

$$
H_{3}:=\langle a, b \mid[[a, b], a]=[[a, b], b]=e\rangle .
$$

All elements of $H_{3}$ can be written in the form $c^{z} b^{y} a^{x}$, with $x, y, z \in \mathbb{Z}$.

We will calculate all the Busemann points of this group with the word length metric coming from the standard generating set $S:=\left\{a, b, a^{-1}, b^{-1}\right\}$. Our work relies on the following formula for this metric, found by Blachère [3].

Theorem 5.1 ([3]). Let $g:=c^{z} b^{y} a^{x}$ be an element of $H_{3}$. Its distance to the identity with respect to the generating set $S:=\left\{a, b, a^{-1}, b^{-1}\right\}$ is

I. if $z y x \geq 0$, and

I.1. if $\max \left(x^{2}, y^{2}\right) \leq|z|$, then

$$
d(e, g)=2\lceil 2 \sqrt{|z|}\rceil-|x|-|y| ;
$$

I.2. if $\max \left(x^{2}, y^{2}\right) \geq|z|$, and

I.2.1. $|x y| \geq|z|$, then

$$
d(e, g)=|x|+|y|
$$

I.2.2. $|x y| \leq|z|$, then

$$
d(e, g)=2\lceil\min (|z / x|,|z / y|)\rceil+|| x|-| y|| ;
$$

II. if $z y x \leq 0$, and

II.1. if $\max \left(x^{2}, y^{2}\right) \leq|z|+|x y|$, then

$$
d(e, g)=2\lceil 2 \sqrt{|z|+|x y|}\rceil-|x|-|y| ;
$$

II.2. if $\max \left(x^{2}, y^{2}\right) \geq|z|+|x y|$, then

$$
d(e, g)=2\lceil\min (|z / x|,|z / y|)\rceil+|x|+|y| .
$$


Given a word $y$, we call the pairs of consecutive letters of $y$ the transitions of $y$. The transitions $a b, b a^{-1}, a^{-1} b^{-1}$, and $b^{-1} a$ are said to be positive. The transitions obtained by reversing the letters of these are called negative. Observe that the group element corresponding to a positive transition is equal to $c$ times the element corresponding to the negative transition obtained by reversing the order of the letters. So, if one takes a word and transforms it by reversing an equal number of positive and negative transitions, the resulting word is a geodesic if and only if the original was.

Proposition 5.2. Every infinite geodesic word in $\mathrm{H}_{3}$ either has two elements of $\mathrm{S}$ as letters or consists of a finite prefix followed by a single letter repeated.

Proof. Let $y$ be an infinite word that has at least three elements of $S$ as letters, at least two of which appear infinitely often.

We wish to show that $y$ is not a geodesic. Obviously, this is the case if it is not freely reduced, so we assume the contrary. Our above assumptions on $y$ then imply that there are either infinitely many positive transitions in $y$ or there are infinitely many negative transitions.

Since $y$ has at least three elements of $S$ as letters, it must have as letters both some generator and its inverse. By repeatedly swapping this generator with one of its neighbours, we can bring it to a position adjacent to its inverse. If $y$ has both infinitely many positive and infinitely many negative transitions, then we can compensate for these swaps by reversing transitions elsewhere so that the total number of positive transitions reversed equals the total number of negative ones reversed. Since the resulting word is clearly not a geodesic, it follows that $y$ is not either.

So we will assume without loss of generality that there are infinitely many positive transitions and finitely many negative ones. Therefore $y$ can be written in the form

$$
y=R a^{i_{0}} b^{j_{0}} a^{-i_{1}} b^{-j_{1}} a^{i_{2}} \ldots,
$$

where $\left(i_{n}\right)$ and $\left(j_{n}\right)$ are sequences of positive integers, and $R$ is some finite initial word.

Consider what happens if $\left(i_{n}\right)$ and $\left(j_{n}\right)$ eventually become, respectively, constants $i$ and $j$. Define the word $w:=a^{i} b^{j} a^{-i} b^{-j}$. Observe that $\bar{w}=c^{i j}$. So, the word $w w w \ldots$ is not geodesic since, by the formula of Theorem 5.1, $d\left(e, \bar{w}^{n}\right)$ grows like the square root of $n$, rather than linearly. Therefore, in this case, $y$ is not geodesic.

If $\left(i_{n}\right)$ and $\left(j_{n}\right)$ are not both eventually constant, then we can find $n \geq 1$ such that $i_{n}<i_{n+1}$ or $j_{n}<j_{n+1}$. Assume that the latter holds; the former case can be handled similarly. Also assume without loss of generality that $n$ is even. So $y$ can be written

$$
y=R \ldots b^{-j_{n-1}}\left(a^{i_{n}}\right) b^{j_{n}} a^{-i_{n+1}} b^{-j_{n+1}} \ldots .
$$

By moving the bracketed clump of $a$ 's forward, we get a word

$$
z=R \ldots b^{-j_{n-1}} b^{j_{n}} a^{-i_{n+1}} b^{-j_{n}}\left(a^{i_{n}}\right) b^{-j_{n+1}+j_{n}} \ldots,
$$


which is clearly not a geodesic. However, $z$ is obtained from $y$ by reversing the letters of $i_{n} j_{n}$ positive transitions and then reversing the letters of the same number of negative transitions. It follows that $y$ is not geodesic either.

Theorem 5.3. Let $\epsilon_{a}$ and $\epsilon_{b}$ be in $\{-1,+1\}$, and let $\Omega$ be the set of all words having letters in $\left\{a^{\epsilon_{a}}, b^{\epsilon_{b}}\right\}$ with both letters occurring infinitely often. Then, all words in $\Omega$ are geodesic, and the corresponding paths all converge to the same Busemann point, given by

$$
\xi^{\epsilon_{a} \epsilon_{b}}\left(c^{z} b^{y} a^{x}\right):=-\epsilon_{a} x-\epsilon_{b} y .
$$

Proof. Let $w$ be a finite word having set of letters $\left\{a^{\epsilon_{a}}, b^{\epsilon_{b}}\right\}$, and let $\bar{w}=c^{z} b^{y} a^{x}$ be the associated element of the group. If $z \neq 0$, then $\operatorname{sign} z=\operatorname{sign} y$. $\operatorname{sign} x=\epsilon_{b} \epsilon_{a}$. Therefore, $z y x \geq 0$. Also, $|z| \leq|x y|$. We deduce from the word length formula of Theorem 5.1 that $d(e, \bar{w})=|x|+|y|$, which is exactly the number of letters in $w$. So $w$ is geodesic. It follows that every word in $\Omega$ is geodesic.

Let $w_{1}$ and $w_{2}$ be words in $\Omega$, and let $\gamma_{1}$ and $\gamma_{2}$ be the corresponding geodesic paths starting at the identity. Choose $N \in \mathbb{N}$, and let $u$ be the prefix of $w_{1}$ of length $N$. Since $w_{2}$ has each of the letters $a^{\epsilon_{a}}$ and $b^{\epsilon_{b}}$ infinitely often, we can find a prefix $v$ of $w_{2}$ that has more $a^{\epsilon_{a}}$ 's and $b^{\epsilon_{b}}$ 's than $u$. So there exists a word $x$ with letters in $\left\{a^{\epsilon_{a}}, b^{\epsilon_{b}}\right\}$ of length $|v|-|u|$ such that $\bar{u} \bar{x}=c^{m} \bar{v}$ for some $m \in \mathbb{Z}$. Since $w_{2}$ has infinitely many positive transitions and infinitely many negative transitions, we can write it $w_{2}=v y \omega$, where $\omega$ is an infinite word (in $\Omega$ ) and $y$ is a finite word satisfying $\bar{y}=c^{m} \bar{z}$, for some $z$ with letters in $\left\{a^{\epsilon_{a}}, b^{\epsilon_{b}}\right\}$. By the first part of the proposition, $u x z \omega$ is a geodesic word. The corresponding geodesic path agrees with $\gamma_{1}$ up to time $N$ and agrees with $\gamma_{2}$ after time $|u x z|$. A similar argument shows that there is also a geodesic path agreeing with $\gamma_{2}$ up to time $N$ and eventually coinciding with $\gamma_{1}$. We now apply Proposition 2.1 to deduce that $\gamma_{1}$ and $\gamma_{2}$ converge to the same limit.

Consider the sequence $h_{n}:=\left(a^{\epsilon_{a}} b^{\epsilon_{b}}\right)^{n}$. By the previous part of the proposition, this sequence converges to the common limit of the geodesics in $\Omega$. We have

$$
h_{n}=c^{\epsilon_{a} \epsilon_{b} n(n+1) / 2} b^{\epsilon_{b} n} a^{\epsilon_{a} n} .
$$

Let $g:=c^{z} b^{y} a^{x}$ be an arbitrary point in $H_{3}$. We have

$$
d\left(g, h_{n}\right)=d\left(e, g^{-1} h_{n}\right)=d\left(e, c^{y x-z-\epsilon_{b} x n+\epsilon_{a} \epsilon_{b} n(n+1) / 2} b^{\epsilon_{b} n-y} a^{\epsilon_{a} n-x}\right) .
$$

Consider what happens when $n$ is large. The exponents of $c, b$, and $a$ will have the signs $\epsilon_{a} \epsilon_{b}, \epsilon_{b}$, and $\epsilon_{a}$, respectively; therefore, their product will be positive. The square of the exponents of both $a$ and $b$ will be approximately twice the absolute value of that of $c$, as will the absolute value of the product of the exponents of $a$ and $b$. Looking at the formula of Theorem 5.1, we see that, for large $n$, the relevant case is I.2.1, and so $d\left(g, h_{n}\right)=\left|\epsilon_{b} n-y\right|+\left|\epsilon_{a} n-x\right|$. In particular $d\left(e, h_{n}\right)=2 n$. So the limiting horofunction is $\xi^{\epsilon_{a} \epsilon_{b}}\left(c^{z} b^{y} a^{x}\right):=-\epsilon_{a} x-\epsilon_{b} y$. 
Theorem 5.4. The following functions are Busemann points of $H_{3}$ :

$$
\begin{aligned}
\eta_{m, n}^{\epsilon}\left(c^{k} b^{j} a^{i}\right):= & -\epsilon i+|j-n|-|n| \\
& +2 J(\epsilon(j-n),(j-n) i-(k-m))-2 J(-\epsilon n, m), \\
\zeta_{m, l}^{\epsilon}\left(c^{k} b^{j} a^{i}\right):= & -\epsilon j+|i-l|-|l| \\
& +2 J(-\epsilon(i-l), j l-(k-m))-2 J(\epsilon l, m),
\end{aligned}
$$

where $\epsilon \in\{-1,+1\}, m, n, l \in \mathbb{Z}$, and

$$
J(u, v):=\left\{\begin{array}{ll}
1, & \text { if } v \neq 0 \text { and } u v \geq 0, \\
0, & \text { otherwise, }
\end{array} \quad \text { for } u, v \in \mathbb{Z} .\right.
$$

Together with $\xi^{++}, \xi^{-+}, \xi^{++}$, and $\xi^{--}$, these are the only Busemann points.

Proof. Choose $\epsilon \in\{-1,+1\}$ and $m, n \in \mathbb{Z}$, and define the path $\gamma_{m, n}^{\epsilon}(t):=c^{m} b^{n} a^{\epsilon t}$, for $t \in \mathbb{Z}$. By the formula of Theorem 5.1,

$$
d\left(\gamma_{m, n}^{\epsilon}(0), \gamma_{m, n}^{\epsilon}(t)\right)=d\left(e, a^{\epsilon t}\right)=t .
$$

Therefore $\gamma_{m, n}^{\epsilon}$ is a geodesic starting at $c^{m} b^{n}$.

Let $g:=c^{k} b^{j} a^{i}$ be an element of $H_{3}$. We have

$$
d\left(g, \gamma_{m, n}^{\epsilon}(t)\right)=d\left(e, c^{m-k+(j-n) i} b^{n-j} a^{\epsilon t-i}\right) .
$$

Write

$$
x_{t}:=\epsilon t-i, \quad y:=n-j, \quad \text { and } \quad z:=m-k+(j-n) i .
$$

So $\left|x_{t}\right|$ tends to infinity as $t$ tends to infinity, whereas $y$ and $z$ remain constant.

Therefore the only relevant cases in the formula of Theorem 5.1 when $t$ is large are I.2.1. and II.2. If $z=0$, then both cases give the same result: $\left|x_{t}\right|+|y|$. If $z \neq 0$, then we have case I.2.1 if $\epsilon y z>0$, in which case $d\left(g, \gamma_{m, n}^{\epsilon}(t)\right)=\left|x_{t}\right|+|y|$, or case II.2. if $\epsilon y z \leq 0$, which gives $d\left(g, \gamma_{m, n}^{\epsilon}(t)\right)=\left|x_{t}\right|+|y|+2$, for $x_{t}$ large enough. To sum up:

$$
d\left(g, \gamma_{m, n}^{\epsilon}(t)\right)=\left|x_{t}\right|+|y|+2 J(-\epsilon y, z) .
$$

Subtracting from this the value obtained when $i=j=k=0$, and taking the limit as $t$ tends to infinity, we see that the path $\gamma_{m, n}^{\epsilon}$ converges to the horofunction $\eta_{m, n}^{\epsilon}$ defined above. This horofunction is a Busemann point since $\gamma_{m, n}^{\epsilon}$ is a geodesic.

Similar reasoning shows that the path $\lambda_{m, l}^{\epsilon}(t):=c^{m+\epsilon t l} b^{\epsilon t} a^{l}$ converges to $\zeta_{m, l}^{\epsilon}$, for all $\epsilon \in\{-1,+1\}$ and $m, l \in \mathbb{Z}$, and that the limit is a Busemann point.

By Proposition 5.2, every infinite geodesic in $H_{3}$ either uses exactly two generators or uses all but one only a finite number of times. In the former case, the geodesic converges to one of $\xi^{++}, \xi^{-+}, \xi^{+-}$, and $\xi^{--}$, by Theorem 5.3. In the latter, the geodesic must eventually coincide with either $\gamma_{m, n}^{\epsilon}$ or $\lambda_{m, l}^{\epsilon}$ for some value of $\epsilon$ and of $m$ and $n$, or $m$ and $l$. Therefore, in this case, the geodesic converges to the corresponding $\eta_{m, n}^{\epsilon}$ or $\zeta_{m, l}^{\epsilon}$. 
The action of $H_{3}$ on each of the Busemann points can easily be calculated:

$$
\begin{aligned}
\left(c^{z} b^{y} a^{x}\right) \eta_{m, n}^{\epsilon} & =\eta_{m+z+n x, n+y}^{\epsilon}, \\
\left(c^{z} b^{y} a^{x}\right) \xi_{m, l}^{\epsilon} & =\zeta_{m+z-y(l+x), l+x}^{\epsilon}, \\
\left(c^{z} b^{y} a^{x}\right) \xi^{\epsilon_{a} \epsilon_{b}} & =\xi^{\epsilon_{a}, \epsilon_{b}} .
\end{aligned}
$$

So, in particular, each of the Busemann points $\xi^{++}, \xi^{+-}, \xi^{-+}$, and $\xi^{--}$is fixed by the action of $\mathrm{H}_{3}$. We have here an illustration of Theorem 1.1. The abelianisation of $H_{3}$ is isomorphic to $\mathbb{Z}^{2}$. One can take the map $\phi$ to be $\phi\left(c^{z} b^{y} a^{x}\right):=(x, y)$. The image of $S$ under this map is the set $\{(1,0),(0,1),(-1,0),(0,-1)\}$, and so $P$ is the square with these points as corners. Of course, $P$ has four facets, corresponding to the four fixed Busemann points.

In [7], Webster and Winchester conjectured that there is a boundary point or points of the form $\lim _{i \rightarrow \infty} \eta_{m_{i}, n_{i}}^{+}$, where $m_{i}$ and $n_{i}$ grow without bound as $i$ tends to infinity, with $m_{i} \geq \alpha n_{i}$ eventually for any $\alpha$, and that this point or these points are fixed under the action of $H_{3}$. Using the formula for $\eta_{m, n}^{+}$from Theorem 5.4 one can calculate that there is exactly one point of this form, namely $\xi^{++}$, which is indeed a fixed point.

\section{References}

[1] M.Akian, S. Gaubert, and C. Walsh, The max-plus Martin boundary. Doc. Math. 14 (2009), 195-240. Zbl 1182.31017 MR 2538616

[2] W. Ballmann, M. Gromov, and V. Schroeder, Manifolds of nonpositive curvature. Progr. Math. 61, Birkhäuser, Boston 1985. Zbl 0591.53001 MR 0823981

[3] S. Blachère, Word distance on the discrete Heisenberg group. Colloq. Math. 95 (2003), 21-36. Zbl 1010.05034 MR 1967551

[4] M. Develin, Cayley compactifications of abelian groups. Ann. Comb. 6 (2002), 295-312. Zbl 1021.05045 MR 1980341

[5] M. Gromov, Hyperbolic manifolds, groups and actions. In Riemann surfaces and related topics: Proceedings of the 1978 Stony Brook Conference (State Univ. New York, Stony Brook, N.Y., 1978), Ann. of Math. Stud. 97, Princeton University Press, Princeton, N.J., 1981, 183-213. Zbl 0467.53035 MR 0624814

[6] M.A. Rieffel, Group $C^{*}$-algebras as compact quantum metric spaces. Doc. Math. 7 (2002), 605-651. Zbl 1031.46082 MR 2015055

[7] C. Webster and A. Winchester, Busemann points of infinite graphs. Trans. Amer. Math. Soc. 358 (2006), 4209-4224. Zbl 1174.46035 MR 2219016

Received October 27, 2008; revised March 2, 2009

C. Walsh, CMAP, Ecole Polytechnique, 91128 Palaiseau Cédex, France

E-mail: cormac.walsh@inria.fr 